\title{
Self-trapping in B-doped amorphous Si: Intrinsic origin of low acceptor efficiency
}

\author{
I. Santos, $,{ }^{1,2}, *$ P. Castrillo, ${ }^{2}$ W. Windl,,${ }^{1}$ D. A. Drabold,${ }^{3}$ L. Pelaz, ${ }^{2}$ and L. A. Marqués ${ }^{2}$ \\ ${ }^{1}$ Department of Materials Science and Engineering, The Ohio State University, 2041 College Road, Columbus, Ohio 43210-1178, USA \\ ${ }^{2}$ Departamento de Electricidad y Electrónica, Universidad de Valladolid, ETSI de Telecomunicación, 47011 Valladolid, Spain \\ ${ }^{3}$ Department of Physics and Astronomy, Ohio University, Athens, Ohio 45701-2979, USA
}

(Received 4 November 2009; revised manuscript received 23 December 2009; published 19 January 2010)

\begin{abstract}
We have used $a b$ initio simulations to study the doping efficiency of amorphous semiconductors, in particular of B-doped amorphous Si. We have found that even in the optimum case of substitutional doping in dangling-bond free amorphous Si the holes provided by B atoms do not behave as free carriers. Instead, they are trapped into regions with locally distorted bond angles. Thus, the effective activation energy for hole conduction turns to be the hole binding energy to these traps. In the case of high B concentration, the trap states move deeper in the gap and the binding energy and spatial localization of holes increase. In addition, B atoms have lower energies for shorter bond lengths, configurations favored in the vicinity of these traps.
\end{abstract}

DOI: 10.1103/PhysRevB.81.033203

PACS number(s): 31.15.A-, 71.55.Jv, 81.05.Gc

The possibility of doping amorphous $\mathrm{Si}(a-\mathrm{Si})$ and hydrogenated amorphous $\mathrm{Si}(a-\mathrm{Si}: \mathrm{H})$ and their lower production cost with respect to crystalline $\mathrm{Si}(c-\mathrm{Si})$ have made them the materials of choice for many different applications in optoelectronics and photovoltaics. ${ }^{1}$ Nevertheless, these materials have an unexpected low doping efficiency, ${ }^{2}$ which limits their use in device applications.

Doping in amorphous materials poses various interesting questions not encountered in crystals. In tetrahedral amorphous semiconductors, dopants are thought to become electrically active when they are in a fourfold configuration, known as substitutional doping, and thus they can provide the material with a carrier. ${ }^{3}$ However, relaxation effects in the amorphous matrix can be substantial after the incorporation of dopants so that it is not obvious a priori whether they will end in a doping configuration or not. ${ }^{2}$ In addition, amorphous networks have tail states in the valence and conduction bands (usually of exponential "Urbach" form) that may be localized or extended (albeit with poor connectivity) in space, ${ }^{4}$ as well as localized midgap states commonly associated to dangling bonds (which are induced by threefold coordinated $\mathrm{Si}$ atoms). ${ }^{2}$ Experiments indicate that the majority of the excess carriers introduced by dopants does not occupy shallow tail states as it would be expected in an ideal intrinsic material but rather midgap states. ${ }^{2}$ In addition, it has been also found that the carrier mobility is degraded and the activation energy for conduction increases when increasing the dopant concentration, ${ }^{5}$ findings that are characteristic of highly localized trapping states. As a consequence, the doping efficiency is reduced.

Several plausible arguments have been developed for explaining these observations. Some authors proposed that dopants induce midgap states, ${ }^{2,5}$ while others, based on experiments $^{6}$ and subsequent calculations, ${ }^{7}$ argued that $\mathrm{H}$ passivation plays an important role in $a-\mathrm{Si}: \mathrm{H}$, the material of interest for photovoltaic applications rather than pure $a$-Si. In both arguments the low doping efficiency is attributed to the influence of external factors, namely, dopants or $\mathrm{H}$ atoms. Apart from these widely accepted factors, there are indications that carrier traps can also be generated by highly distorted bonds. Using tight-binding calculations Bagolini et $a{ }^{8}{ }^{8}$ found that distorted bond angles can localize elec- tronic states in $a$-Si. Using ab initio calculations Wagner et $a l .{ }^{9}$ found that optical excitations can generate distorted bond angles in $a-\mathrm{Si}$, which act as hole traps. In addition, other theoretical works found that sufficiently distorted bond angles generate midgap levels rather than shallow levels without the intervention of dangling bonds or floating bonds (which are induced by fivefold Si atoms). ${ }^{10}$ Nevertheless, the present understanding of how these traps may influence the free-carrier concentration is rather incomplete.

In this work we have used $a b$ initio simulations to address the analysis of the low doping efficiency of B-doped $a$-Si, the main $p$-type dopant in $\mathrm{Si}$. We focus our study on substitutional $\mathrm{B}$ doping of dangling bond free pure $a$-Si. This can be considered the most ideal situation not influenced by any external factor and the limit case for an optimal technological matrix. In this scenario, we analyze the spatial localization of the holes introduced by $\mathrm{B}$ atoms, and the electronic density of states (EDOS) of the resulting doped material in order to evaluate the possible role of intrinsic features of the amorphous matrix in controlling the free hole concentration.

For this Brief Report we employ two different cubic $a-\mathrm{Si}$ cells containing 64 and 216 atoms, which are generated using the procedure proposed by Barkema and Mousseau. ${ }^{11}$ The calculations presented in this work are referred to the 64-atom cell, while the 216-atom cell is used to test the obtained findings in a larger simulation cell. Periodic boundary conditions are employed in all spatial directions. We use the density-functional theory code VASP (Refs. 12 and 13) with generalized gradient approximation ultrasoft pseudopotentials. ${ }^{14}$ The energy cutoff chosen is $230 \mathrm{eV}$ in all calculations. We use a $4 \times 4 \times 4$ Monkhorst-Pack $k$-point mesh for EDOS calculations in the 64-atom cell, and $2 \times 2$ $\times 2$ for cell relaxations, and also for EDOS calculations in the 216-atom cell. Although the amorphous simulation cells are generated externally to VASP, they barely change at all when they are relaxed with $a b$ initio interactions. After this relaxation all the $\mathrm{Si}$ atoms in the amorphous cells are fourfold coordinated, i.e., there are neither dangling nor floating bonds. While the atomic bond lengths and bond angles in $c$-Si are $d_{c-\mathrm{Si}}=2.36 \AA$ and $\theta_{c-\mathrm{Si}}=109.47^{\circ}$, the corresponding averaged values in the $a$-Si cells used are $d_{a \text {-Si }}$ $=2.37 \pm 0.08 \AA$, and $\theta_{a-\mathrm{Si}}=109 \pm 10^{\circ}$, respectively. These 


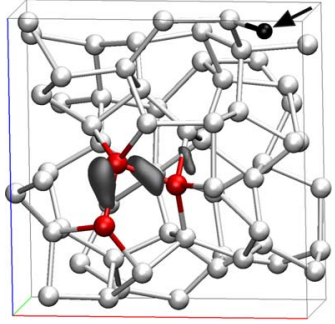

a) $\left(\mathrm{a}-\mathrm{Si}_{63} \mathrm{~B}\right)^{0}$

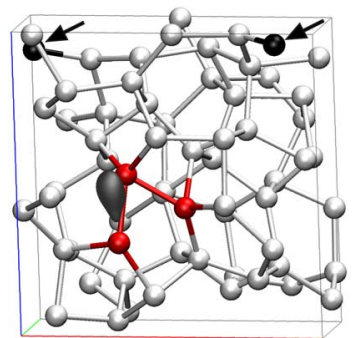

c) $\left(\mathrm{a}-\mathrm{Si}_{62} \mathrm{~B}_{2}\right)^{0}$

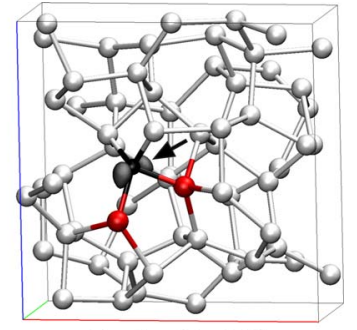

b) $\left(\mathrm{a}-\mathrm{Si}{ }_{63} \mathrm{~B}\right)^{0}$

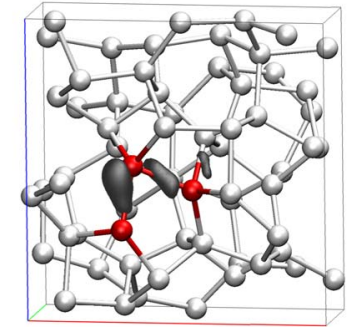

d) $\left(\mathrm{a}-\mathrm{Si}_{64}\right)^{+}$
FIG. 1. (Color online) Hole spatial localization in 64-atom cells with (a) a B atom far from the HT region, (b) a B atom at the HT, and (c) two B atoms far from the HT, (d) as well as in a positively charged undoped cell. The dark shadowed areas show the isosurface at $50 \%$ of the maximum hole density. B atoms are black and marked by arrows. Si atoms are white and red (gray). The red $\mathrm{Si}$ atoms indicate the position of the HT.

values are within the expected range of well-relaxed computer-generated $a$-Si cells. ${ }^{11,15}$

We have considered 64 different B configurations by successively replacing each of the $\mathrm{Si}$ atoms of the 64-atom cell by a B atom. By sampling all 64 sites, we accumulate some reasonable statistics. We performed relaxations of both the atomic positions and cell shape and volume to find the nearest local minimum energy configuration for each replacement. In all the resulting structures, B atoms are fourfold coordinated, and thus, they are expected to provide the sample with one hole. The average $B$ bond length is $2.07 \pm 0.06 \AA$, in agreement with the obtained in $c$-Si of $2.08 \AA$. In the case of an ideal acceptor, the hole would be in a shallow state locally extended around the dopant. ${ }^{16}$ However, this did not happen in our simulations. In Fig. 1 we represent the hole localization for different B configurations in the 64-atom cell. For the configuration shown in Fig. 1(a), the hole is localized far from the B atom. In fact, we found that, independently of the position of the B atom, the hole is localized at the same region of the cell with a similar density distribution. This region is represented in Fig. 1 by the colored $\mathrm{Si}$ atoms, and the hole localization by the shadowed areas. When the B atom is situated at this region [Fig. 1(b)], the hole-localization is even stronger. We have also analyzed the situation where two $\mathrm{Si}$ atoms are replaced by $\mathrm{B}$, representing the case of high dopant concentration. For that purpose, we selected two $\mathrm{Si}$ atomic positions more than $7 \AA$ apart from the red atoms of Fig. 1 and $\sim 5.5 \AA$ from each other. The resulting configuration is shown in Fig. 1(c), together with the spatial localization of the holes provided by $\mathrm{B}$ atoms. As it can be seen, the holes are again at the same zone, and not in the neighborhood of B atoms.
To elucidate whether the hole-localization region is induced by the $\mathrm{B}$ atoms or it exists even in the absence of acceptors, we analyzed the undoped 64-atom cell with positive charge, $\left(a-\mathrm{Si}_{64}\right)^{+}$, i.e., with the same number of electrons than the studied neutral $\mathrm{B}$ configurations, $\left(a-\mathrm{Si}_{63} \mathrm{~B}\right)^{0}$. The resulting hole localization, shown in Fig. 1(d), is very similar to that of Fig. 1(a). We also considered the case of adding two holes to the $a$-Si cell (not shown) and their localization agreed with that shown in Fig. 1(c). Therefore, the region of interest is not induced by the B atoms but it is inherent to the $a$-Si matrix. In this sample we have found that when there are not holes available in the cell [for example, in $\left(a-\mathrm{Si}_{64}\right)^{0}$ or $\left(a-\mathrm{Si}_{63} \mathrm{~B}\right)^{-}$configurations not shown in Fig. 1], some of the bond angles at the hole-localization region are $\$ 90^{\circ}$. When one hole is captured [Figs. 1(a), 1(b), and 1(d)], the bond angles in the colored zigzag become more distorted $\left(<85^{\circ}\right)$, and the distortion is even higher when two holes are captured, with angles $\sim 75^{\circ}$ [for example, in Fig. 1(c)]. Furthermore, one of the $\mathrm{Si}$ atoms involved in the holelocalization region [in particular the $\mathrm{Si}$ atom replaced by $\mathrm{B}$ in Fig. 1(b)] is almost in a "flat configuration" in all the charge states, i.e., with three angles adding $\sim 360^{\circ}$. The relation between distorted bond angles and hole localization agrees with previous studies. ${ }^{8,9}$ Our results also indicate that the hole affinity of these regions is higher than that of B acceptors. Additionally, the bond distortion increases with the number of holes localized on it, independently if they come from $\mathrm{B}$ atoms or from charge modifications in the undoped $a$-Si cell.

We have studied the EDOS of the configurations of Fig. 1, which are represented in Fig. 2. When the B atom is far from the distorted region [Fig. 1(a)], the EDOS [Fig. 2(a)] has a shallow state near the top of the valence band. The position of the Fermi level indicates that the added hole is at the state associated with that peak. The same comment holds true for the case of placing the B atom at the distorted region [Fig. 2(b)]. Nevertheless, the combination of the acceptor nature of the B atom with the hole affinity of the distorted region results in the stronger localization of the hole around the $B$ atom [Fig. 1(b)]. In both of the previous cases there is a good agreement with the EDOS of the $a$-Si cell positively charged (specially when the B atom is far from the trapping region), which also has a peak near the valence-band edge (VBE). When there are two B atoms in the $a$-Si cell [Fig. 1(c)], the EDOS [Fig. 2(c)] shows a midgap peak. Since the Fermi level is at the VBE, the two holes are at the states associated with that peak. A very similar EDOS is obtained for the undoped $a$-Si cell when two holes are added to it. In Fig. 2(d) we compare the EDOS of the neutral undoped $a$-Si cell, with that of a B-doped $a$-Si cell with negative charge [in particular with the B atom at the same position as in Fig. 1(a)], showing a very good agreement. Thus, in all cases the doped and undoped $a$-Si cells with the same total number of electrons have a very similar EDOS.

From these results we conclude that there is a hole trap (HT) in the $a$-Si cell, which is associated with a region with highly distorted bond angles and captures holes provided by $\mathrm{B}$ atoms. Furthermore, its energy level changes its position in the gap according to its occupancy. When the HT is not occupied by holes, its energy level is stuck to the valence 


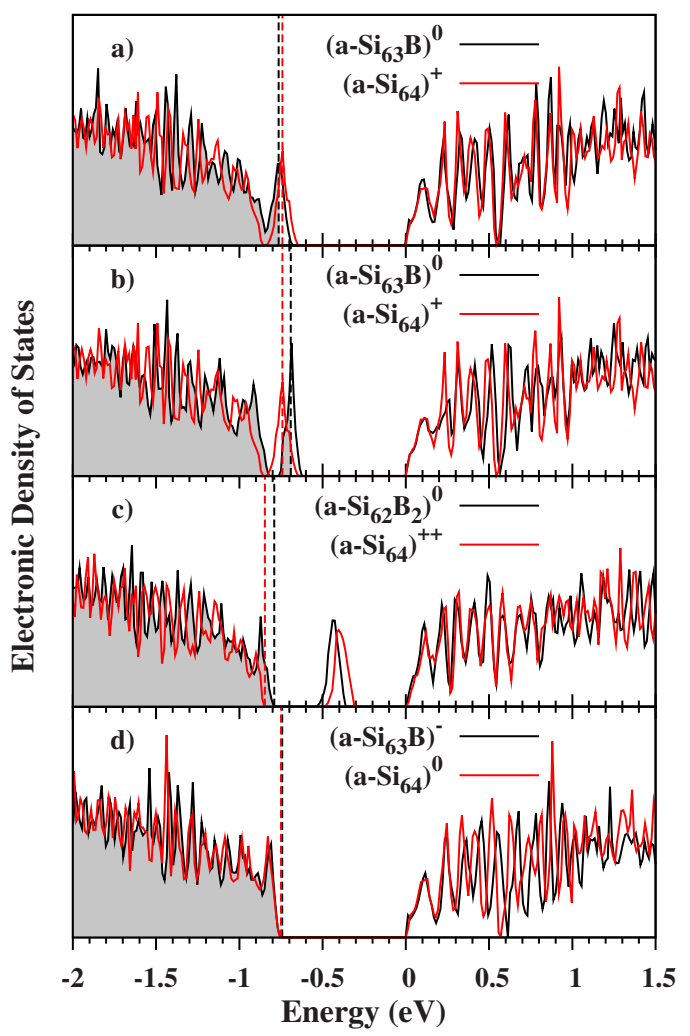

FIG. 2. (Color online) [(a)-(c)] EDOS of the corresponding B configurations of Fig. 1, together with EDOS of the undoped cell with the same number of electrons. (d) EDOS of the neutral undoped cell, together with the EDOS of a B-doped $a$-Si cell with negative charge where the $\mathrm{B}$ atom is at the same position as in Fig. 1(a). The origin of energies is at the conduction-band edge (CBE) for convenience. The Fermi levels are indicated by vertical dashed lines. The shadowed area schematically shows the electronic filling.

band. As one or two holes are captured, the level goes deeper and deeper in the gap. We have checked that, in contrast, the addition of one or two electrons $\left[\left(a-\mathrm{Si}_{64}\right)^{-1}\right.$ and $\left(a-\mathrm{Si}_{63} \mathrm{~B}\right)^{-2}$ cells] produce neither gap states nor relevant changes in the EDOS (not shown).

We have evaluated the hole binding energy, $E_{b}$, for the different changes in the HT occupancy as

$$
\begin{gathered}
E_{b}^{+/ 0}=E_{\mathrm{T}}^{0}-E_{\mathrm{VBE}}^{0}-E_{\mathrm{T}}^{+}, \\
E_{b}^{++/+}=E_{\mathrm{T}}^{+}-E_{\mathrm{VBE}}^{+}-E_{\mathrm{T}}^{++},
\end{gathered}
$$

where $E_{\mathrm{T}}$ is the total energy and $E_{\mathrm{VBE}}$ is the energy of an electron at the VBE when the HT has no holes $\left({ }^{0}\right)$, one $\left({ }^{+}\right)$ or two holes $\left({ }^{++}\right) . E_{b}$ turns to be the effective activation energy for hole conduction. It is noteworthy that, due to atomic rearrangements when adding/removing holes, $E_{b}$ cannot be simply calculated as the energy difference of the EDOS peaks to the VBE. The obtained energies for the undoped cell are $E_{b}^{+/ 0}=0.16 \mathrm{eV}$ and $E_{b}^{++/+}=0.29 \mathrm{eV}$. In $a$-Si cells with one $\mathrm{B}$ atom, the energies are $E_{b}^{+/ 0}$ $=0.15 \pm 0.03 \mathrm{eV}$ and $E_{b}^{++/+}=0.27 \pm 0.07 \mathrm{eV}$, which are comparable with those of the undoped $a$-Si cell. In the case of Fig. 1(c), we found $E_{b}^{+/ 0}=0.13 \mathrm{eV}$ and $E_{b}^{++/+}=0.20 \mathrm{eV}$,

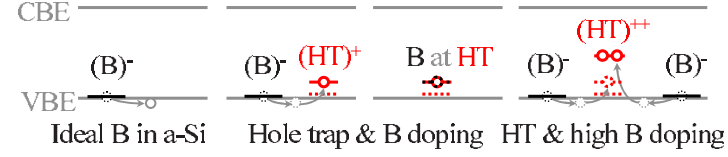

FIG. 3. (Color online) Band scheme showing the effect of the studied HT in the B doping of $a$-Si (see text for details).

which are slightly lower than in the previous cases. This difference might be due to rearrangements when relaxing the cell with two B atoms since they have shorter bond lengths. In any case, $E_{b}$ increases with the occupancy of the HT. The good agreement in $E_{b}$ for the doped and undoped $a$-Si cells indicates that it is controlled by the HT and not by the B atoms.

In the 216-atom cell we also found that holes provided to the sample (by modifying the charge state or by replacing one or two $\mathrm{Si}$ atoms at different positions of the cell by B) were localized in a region associated to highly distorted bond angles, which also acts as a HT. These findings were confirmed by the analysis of the EDOS since the holes were at the states associated with the HT. In addition, we have found that $E_{b}=0.12 \mathrm{eV}$ for the doped and undoped 216-atom cells, which also indicates that the binding energy is controlled by the HT.

According to these results, we schematically show in Fig. 3 the effect of the HT in the B doping of $a$-Si. In an ideal scenario $\mathrm{B}$ atoms would have associated a shallow level near the VBE, and they would release easily their hole to the valence band. However, this does not occur when HTs are present since they capture the holes provided by B atoms. Once a hole is captured, $E_{b}$ increases from some meV (supposing a shallow level for $\mathrm{B}$ atoms) to $\sim 0.15 \mathrm{eV}$. If the $\mathrm{B}$ atom is at the HT, the hole has a stronger spatial localization. For high $\mathrm{B}$ concentration, two holes are trapped and $E_{b}$ is higher, $\sim 0.3 \mathrm{eV}$.

This picture helps to understand the role of HTs on B doping in $a$-Si. B atoms can behave as acceptors in the sense of providing the amorphous matrix with holes, but they are ineffective in doping since the HTs capture the available holes. The other appealing feature is that as holes are captured into these traps, $E_{b}$ increases. Hence, $a$-Si exhibits a "self-trapping" behavior for holes through these HTs, with higher hole affinity when holes are already trapped. This can contribute to increase the activation energy for conduction at high B concentration. ${ }^{5}$

We have also analyzed the energetics of B atoms by calculating their energy, $\varepsilon_{B}$, at the 64 different positions in the 64-atom cell as

$$
\varepsilon_{B}=E_{\text {tot }}\left[\left(a-\mathrm{Si}_{63} \mathrm{~B}\right)^{-}\right]-\frac{63}{64} E_{\mathrm{tot}}\left[\left(a-\mathrm{Si}_{64}\right)^{0}\right] .
$$

We have considered negative charged cells $\left[\left(a-\mathrm{Si}_{63} \mathrm{~B}\right)^{-}\right]$to minimize the distortion induced by the capture of holes at the HT. The results with neutral cells $\left[\left(a-\mathrm{Si}_{63} \mathrm{~B}\right)^{0}\right]$ are qualitatively similar but with higher dispersion. We found that when $\mathrm{B}$ atoms replace $\mathrm{Si}$ atoms with shorter $\mathrm{Si}-\mathrm{Si}$ bonds, the resulting B-Si bonds are shorter and energetically more favorable, as shown in Fig. 4(a). In Fig. 4(b) we show the distance 


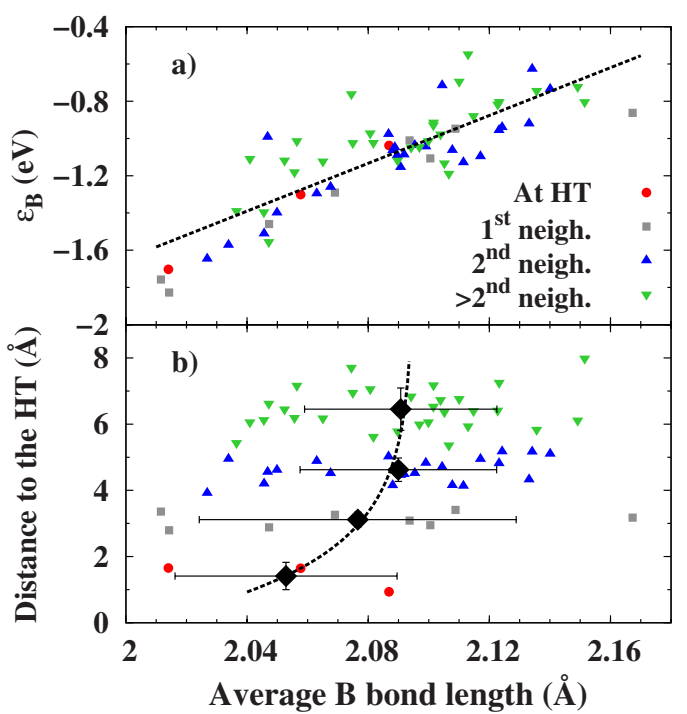

FIG. 4. (Color online) (a) Energy of B atoms as a function of the average $\mathrm{B}$ bond length. The dashed line is a fit to the data. (b) Distance of B atoms to the HT as a function of their average bond length. Diamonds are the average value of the groups, and the dashed line is to guide the eye.

of B atoms to the HT as a function of the average B bond length. We define the HT position as the center of mass of the red atoms of Fig. 1. B atoms placed at the red positions of Fig. 1 are labeled as "At HT," B atoms at the first bondconnected positions as "first neighbor," etc. Diamonds repre- sent the average on the distances of the groups. We found that, despite the scattering of the data, the distortion around the $\mathrm{HT}$ results, on average, in shorter B bond lengths and hence in $\mathrm{B}$ configurations with lower $\varepsilon_{B}$. Then, $\mathrm{B}$ atoms could be retained preferentially near the HT affecting their diffusion through the $a$-Si matrix.

In conclusion, we have found that, even in the most ideal situation of substitutional B doping of fourfold coordinated $a$-Si, the doping efficiency is highly influenced by the presence of HTs in the amorphous matrix associated to distorted bond angles. These HTs capture the available holes. Hence, the effective activation energy for hole conduction is not the ionization energy of the B atom but the binding energy of the hole to the HT. This binding energy depends on the HT occupancy, increasing with the number of trapped holes. The case of two holes at the HT (high B concentration) results in a midgap level without the intervention of dangling bonds. In addition, the distortion induced by the HT results, on average, in shorter B bond lengths at its vicinity, which also results in $\mathrm{B}$ configurations with lower energy.

This work has been partially funded by the Ohio Supercomputer Center (computer time); the Spanish DGI under Grants No. TEC2008-0609 (I.S., L.P., and L.A.M.) and No. TEC2008-05301 (P.C.); the ARO under Grant No. MURI W91NF-06-2-0026 (D.A.D.); and the Ohio State University Center for Emergent Materials (a NSF MRSEC; Grant No. DMR-0820414) (W.W.).

\footnotetext{
*ivasan@tel.uva.es

${ }^{1}$ R. A. Street, Technology and Applications of Amorphous Silicon, Springer Series in Materials Science (Springer-Verlag, Berlin, 2000), Vol. 37.

${ }^{2}$ M. Stutzmann, D. K. Biegelsen, and R. A. Street, Phys. Rev. B 35, 5666 (1987).

${ }^{3}$ R. A. Street, Phys. Rev. Lett. 49, 1187 (1982).

${ }^{4}$ J. Dong and D. A. Drabold, Phys. Rev. Lett. 80, 1928 (1998); Y. Pan, F. Inam, M. Zhang, and D. A. Drabold, ibid. 100, 206403 (2008).

${ }^{5}$ R. A. Street, J. Non-Cryst. Solids 77-78, 1 (1985).

${ }^{6}$ J. B. Boyce and S. E. Ready, Phys. Rev. B 38, 11008 (1988).

${ }^{7}$ P. A. Fedders and D. A. Drabold, Phys. Rev. B 56, 1864 (1997).

${ }^{8}$ L. Bagolini, A. Mattoni, and L. Colombo, Appl. Phys. Lett. 94,
}

053115 (2009)

${ }^{9}$ L. K. Wagner and J. C. Grossman, Phys. Rev. Lett. 101, 265501 (2008).

${ }^{10}$ P. A. Fedders, D. A. Drabold, and S. Klemm, Phys. Rev. B 45, 4048 (1992); P. A. Fedders and D. A. Drabold, ibid. 47, 13277 (1993).

${ }^{11}$ G. T. Barkema and N. Mousseau, Phys. Rev. B 62, 4985 (2000).

${ }^{12}$ G. Kresse and J. Hafner, Phys. Rev. B 49, 14251 (1994).

${ }^{13}$ G. Kresse and J. Furthmüller, Comput. Mater. Sci. 6, 15 (1996); Phys. Rev. B 54, 11169 (1996).

${ }^{14}$ J. P. Perdew and Y. Wang, Phys. Rev. B 45, 13244 (1992).

${ }^{15}$ F. Wooten, K. Winer, and D. Weaire, Phys. Rev. Lett. 54, 1392 (1985).

${ }^{16}$ L.-W. Wang, J. Appl. Phys. 105, 123712 (2009). 